\title{
Politique
}

\section{La politique « africaine " du Québec, de 1960 à 1984}

\section{Myriam Gervais}

Numéro 7, hiver 1985

Projection internationale du Québec

URI : https://id.erudit.org/iderudit/040478ar

DOI : https://doi.org/10.7202/040478ar

Aller au sommaire du numéro

Éditeur(s)

Société québécoise de science politique

ISSN

0711-608X (imprimé)

1918-6584 (numérique)

Découvrir la revue

Citer cet article

Gervais, M. (1985). La politique « africaine » du Québec, de 1960 à 1984.

Politique, (7), 53-66. https://doi.org/10.7202/040478ar d'utilisation que vous pouvez consulter en ligne.

https://apropos.erudit.org/fr/usagers/politique-dutilisation/ 


\title{
La politique «africaine» du Québec, de 1960 à 1984
}

\author{
Myriam Gervais \\ Université du Québec à Montréal
}

Dans sa recherche d'une reconnaissance sur la scène internationale, le Québec a développé des liens avec la France et avec plusieurs pays d'Afrique francophone. Si les relations avec la France ont été abondamment décrites et analysées, peu d'auteurs ont mené des études similaires des activités du Québec dans le continent africain. ${ }^{1}$

Jusqu’à présent, le volet des relations économiques du Québec avec cette région du monde n’a jamais été sérieusement abordé. Les travaux qui en font mention se contentent d'affirmer que les

1. Certains faits ou évènements ayant trait aux relations du Québec avec l'Afrique ont été relatés dans le cadre d'études générales portant sur l'ensemble des relations internationales du Québec. À titre indicatif, nous mentionnons les ouvrages les plus importants: Louise Beaudoin. "Origines et développement du rôle international du gouvernement du Québec." in Paul Painchaud (sous la direction de). Le Canada et le Québec sur la scène internationale. Montréal, PUQ/CQRI, 1977; Richard Gagnon. "Le Québec dans le monde." mémoire de maîtrise, Université d'Ottawa, Ottawa, 1979; Denis Vaugeois. "La coopération du Québec avec l'extérieur."Études Internationales. Vol. V, no. 2, mars 1974; Louis Sabourin. "Canada and Francophone Africa." in Peyton V. Lyon and Tarq Y. Ismael. eds. Canada and the Third World. Toronto, MacMillan, 1975. 
échanges commerciaux avec l'Afrique sont marginaux, voire dérisoires. ${ }^{2}$ Devant le caractère peu fouillé de cette argumentation et la faiblesse des critères utilisés, nous avons cherché à vérifier si cette évaluation sommaire était valable pour l'ensemble de la période 1970-1981 en procédant à une analyse statistique du commerce international du Québec avec l'Afrique. Cette vérification empirique permet de saisir la position relative de cette région par rapport aux autres partenaires commerciaux ainsi qu'à établir la part du Québec dans le développement des relations commerciales du Canada avec l'Afrique. L'intérêt de traiter cet aspect se trouve d'autant plus justifié si l'on fait référence à la politique de promotion des exportations québécoises.

\section{1 - Objectifs sous-tendant le développement des relations du Québec avec l'Afrique}

Il s'avère nécessaire de rappeler les objectifs qui ont présidé aux actions du Québec et du Canada en Afrique car il semble subsister une certaine ambiguité quant aux motivations qui ont déterminé la présence de ces deux acteurs dans cette partie du monde.

La recherche d'une reconnaissance formelle comme entité sur la scène internationale est à l'origine des premières incursions

2. En général, on utilise comme indicateur la part de l'Afrique dans les échanges commerciaux internationaux du Québec pour une ou deux années de référence. Compte tenu de l'importance des États-Unis dans ces échanges, toute autre région du monde ne semble avoir qu'un rôle secondaire. De plus, on ne peut guère en prenant deux points de référence vérifier si l'augmentation ou la diminution du volume des échanges avec l'Afrique repose sur des causes conjoncturelles ou structurelles. 
du Québec en Afrique francophone. ${ }^{3}$ Cette motivation de la part du Québec fut la cause première de l'intérêt du Canada à l'égard de cette région d'Afrique.

Les chercheurs sont unanimes à suggérer que ce sont des motifs d'ordre politique qui prévalent pour expliquer la présence actuelle des deux niveaux de gouvernement dans cette aire géographique. Ainsi l'étude de Linda Freeman impute:

"the remarkable expansion of Canada's program of development assistance to francophone Africa in the 1970s has been part of the federal government's response to the challenge which the Quebec government has issued to its role as the primary actor in Canada's relationships with francophone countries. ${ }^{4}$ "

Pour notre part, si les origines des relations du Canada et du Québec avec l'Afrique francophone ont été influencées par le contentieux Ottawa-Québec en matière de politique étrangère, les raisons d'être de ces relations sont devenues, avec l'évolution de ce dossier et des réalités économiques, plus pragmatiques et font référence à une rationalité nouvelle. Comme nous le verrons dans la période 1978-1984, le Québec et le Canada ont élaboré une politique commerciale mettant l'emphase sur la diversification de leurs partenaires commerciaux afin d'être moins tributaires des soubresauts de l'économie mondiale et des mesures protectionnistes de leurs marchés traditionnels. Cette actualisation des politiques canadienne et québécoise de diversification s'adresse particulièrement aux pays en développement et par voie de conséquence à l'Afrique.

3. En Afrique, les relations politiques et la coopération bilatérale du Québec se concentrent au Maghreb et en Afrique occidentale et centrale francophone. Ce n'est que tout récemment que des contacts indirects ont été établis avec l'Afrique lusophone par le biais de l'Agence de coopération technique et culturelle (A.C.C.T.).

4. Linda Freeman. "Canada and Africa in the 1970's." International Journal. Vol. 35 , no. 4 , aut. 1980 , p. 802 . 


\section{2 - Première phase: $1960-1971$}

Les relations politiques du Québec avec l'Afrique dans les années 60 ont connu un début prometteur, mais furent rapidement annihilées par les pressions exercées par le gouvernement fédéral auprès des pays d'expression francophone.

Une première fois à Libreville lors de la conférence des Ministres de l'Éducation (C.M.E.), puis à Niamey où le Québec a obtenu un statut de gouvernement participant à l'Agence de coopération culturelle et technique (A.C.C.T.), le Québec a été reconnu comme entité par les pays d'Afrique francophone. Par la suite, toutefois, avec la venue au pouvoir du gouvernement Trudeau, le Québec a vu sa marge de manœuvre sur la scène internationale considérablement réduite. En effet, Ottawa a réagi avec vigueur pour endiguer les initiatives du Québec et intégrer celui-ci au sein de la structure fédérale. ${ }^{5}$

Durant cette période, le Québec a conclu de modestes accords de coopération bilatérale et donné suite à diverses propositions d'échanges. Ainsi, on peut mentionner en 1967 l'accord culturel avec le Rwanda et une entente de coopération bilatérale en éducation avec le Gabon, paraphée en 1969.

\section{3 - Deuxième phase: 1971-1978}

Les efforts du Québec pour établir des relations politiques avec des pays africains étant systématiquement contrés par le gouvernement fédéral ${ }^{6}$, le domaine de la coopération a été le

5. C'est dans ce contexte qu'eut lieu la mission Chevrier, laquelle avait pour but de développer tout azimut le financement canadien de projets en Afrique francophone, rendant ainsi tangible une présence canadienne jusque là symbolique et peu manifeste. Cet accroissement de la coopération canadienne devait faire contrepoids à l'action québécoise et placer la reconnaissance du Québec par les pays africains dans une perspective nouvelle. La suite des évènements a confirmé le succès de la stragégie du gouvernement fédéral.

6. C'est dans cette optique qu'il faut interprété le scénario observé lors des négociations entre les deux palliers de gouvernement pour ouvrir une délégation générale 
champ d'intervention privilégié pour assurer une présence québécoise dans la région.

L'action bilatérale du gouvernement s'est surtout caractérisée par l'octroi de bourses aux étudiants africains, par l'accueil de stagiaires dans les domaines de l'éducation et de la santé et par des ententes avec certains pays d'Afrique pour assurer la gratuité des frais scolaires à leurs ressortissants. ${ }^{7}$

Mais devant l'augmentation considérable de l'aide canadienne au pays d'Afrique francophone, ${ }^{8}$ le Québec s'est vu contraint de chercher d'autres avenues que la coopération bilatérale pour maintenir ses relations avec ce continent, ne pouvant rivaliser sur le plan financier avec l'ACDI.

Aussi le Québec a-t-il, bien que cela semble paradoxal, axé l'essentiel de son intervention dans le cadre de la programmation de l'ACDI. Sa collaboration avec cet organisme s'est faite sous la

du Québec à Dakar en 1977. La seule représentation québécoise en Afrique est un délégué du gouvernement qui agit à titre de conseiller en éducation au sein de l'ambassade canadienne d'Abidjan.

7. Les principaux pays bénéficiaires furent: la Cameroun, la Côte d'Ivoire, le Maroc, la Tunisie, le Sénégal et le Togo. (Source: Ministère des Affaires intergouvernementales. Rapport annuel. $1971 / 72$ et ss.).

Pour une analyse détaillée des différentes interventions du Québec dans le domaine de la coopération bilatérale, voir: Myriam Gervais. "Contribution à l'étude des relations internationales du Québec: le cas de l'Afrique." note de recherche, département de science politique, UQAM, Montréal, 1984, $47 \mathrm{p}$.

8. Les allocations pour cette région sont passées de $1 / 2$ million de dollars en 1962 à 6 millions en 1966, 12 millions en 1968 et 32 millions en 1970, soit immédiatement après la mission Chevrier. Pour bien saisir la croissance exceptionnelle de l'Afrique francophone, on peut la comparer à celle de l'Afrique du Commonwealth 51 millions; de 1970 à 1972, les chiffres étaient respectivement de 134 et 129 millions. Depuis lors, une certaine égalité a été maintenue entre les montants accordés à ces deux régions. (Sources: Canada, ACDI. Rapport annuel de l'ACDI. 1967/68. et ss. Ottawa, Information Canada.; Canada, ACDI. «Stratégie de coopération au développement international. 19751980.» Information Canada, Ottawa, 1975, 53 p.) 
forme de projets conjoints où le Québec a agi à titre de maître d'œuvre. ${ }^{9}$ Les principaux projets où le Québec a pris une part active dans les années 70 sont: les projets de santé au Sénégal, d'éducation au Maroc et au Bénin, et de l'hôtellerie en Côte d'Ivoire.

Pour pallier à sa faiblesse financière en matière d'aide, le Québec a privilégié cette formule de coopération dans un souci de «visibilité». S'impliquant dans des projets d'envergure, le Québec, grâce à son apport en ressources humaines sur le terrain, espérait préserver son image et rendre plus tangible sa présence dans la région. ${ }^{10}$ Le Canada a favorisé les programmes conjoints avec le Québec car d'une part, il réinsérait les initiatives québécoises au sein de la structure fédérale et, d'autre part, il avait besoin de son expertise dans certains domaines (éducation, santé, foresterie) ou de son personnel francophone.

Dans l'ensemble, la coopération bilatérale du Québec avec l'Afrique est demeurée modeste: en 1978, le budget de coopération était de l'ordre de 6 millions de dollars. Toutefois, $88.3 \%$ de ce montant était remboursable par l'ACDI dans le cadre des programmes conjoints.

\section{4 - Troisième phase: 1978-1984}

Depuis 1978, les économies occidentales ont connu une crise majeure, caractérisée principalement par un important phénomène d'inflation et une profonde récession. L'économie québécoise a été durement touchée par les effets de cette crise et par les changements

9. Cette formule de coopération n'implique le Québec qu'au niveau de la gestion: il est chargé d'assurer la mise en ouvre et l'exécution des différentes phases du projet. Il ne participe ni à l'orientation ni à l'élaboration des projets sélectionnés. L'entente de principe établi entre les pays donateurs et bénéficiaires est faite par le gouvernement fédéral.

10. Mais ce type d'intervention ne permet ni de se doter d'une politique propre, ni d'acquérir une reconnaissance officielle en tant qu'interlocuteur auprès des gouvernements étrangers, ni d'être perçu comme pays donateur. 
structuraux qui ont affecté la configuration internationale. ${ }^{11} \mathrm{La}$ montée des nouveaux pays industrialisés (NPI), leur concurrence et leur compétivité affaiblissent les marchés traditionnels d'exportation du Québec. Ces contraintes nouvelles revêtent un caractère d'autant plus crucial lorsqu'on considère que le Québec est fortement tributaire, tout comme le Canada, de ses performances sur les marches internationaux pour assurer la croissance de son économie.

Ces modifications au niveau du commerce international ainsi que les mesures protectionnistes qu'elles ont provoquées de la part de plusieurs pays industrialisés, ont incité les gouvernements canadien et québécois à élaborer, quoique séparément, une politique commerciale qui cherche à: 1) renforcer la stabilité des marchés actuels; 2) pénétrer les marchés des pays en développement à revenus moyens. ${ }^{12}$

La création d'un ministère du Commerce extérieur, en janvier 1983, suivit par la restructuration d'avril 1984 qui créa le ministère du Commerce extérieur et des relations internationales, traduisent de façon évidente et concrète la volonté du Québec de faire du commerce extérieur un instrument privilégié du développement de l'économie. Cette prioritisation des échanges économiques dans la stratégie de relance du gouvernement ne peut qu'avoir une forte incidence sur l'orientation des relations internationales du Québec. Aussi, peut-on à juste titre se demander si cette tentative

11. La structure de l'économie mondiale est en pleine mutation. On observe une redistribution de la puissance industrielle: les États-Unis voient leur pouvoir diminuer au profit du Japon, de l'Europe et des NPI. (Les technologies courantes sont transférées vers les NPI, lesquels abaissent les coûts de revient et par conséquent deviennent de plus en plus compétitifs sur le marché mondial.)

12. Ministère du Commerce extérieur. Le commerce international du Québec. Éditeur officiel, Janvier 1984; La politique commerciale du Canada pour les années 80. (document de travail), gouvernement du Canada, août 1983. 


\section{TABLEAU 1}

Répartition des exportations et des importations du Québec selon leur destination et provenance (par grandes régions) pour les années 1972, 1975, 1978 et 1981 .

(en pourcentage)

\begin{tabular}{|l|r|r|r|r||r|r|r|r|}
\hline & \multicolumn{4}{|c||}{ Exportations } & \multicolumn{4}{c|}{ Importations } \\
\cline { 2 - 8 } & 1972 & 1975 & 1978 & 1981 & 1972 & 1975 & 1978 & 1981 \\
\hline Amérique du Nord & 64.0 & 60.4 & 65.1 & 65.0 & n.d. & 43.0 & 45.8 & 46.4 \\
Europe occidentale & 22.6 & 23.4 & 17.2 & 18.0 & n.d. & 19.2 & 21.6 & 18.7 \\
Europe orientale & 0.8 & 0.9 & 1.9 & 0.8 & n.d. & 1.2 & 1.3 & 1.0 \\
Moyen-Orient & 1.0 & 2.2 & 1.8 & 1.4 & n.d. & 12.4 & 5.2 & 4.1 \\
Pays d'Afrique & 1.5 & 1.8 & 1.6 & 3.2 & n.d. & 3.8 & 3.3 & 4.2 \\
Pays d'Asie & 3.6 & 4.3 & 6.5 & 4.7 & n.d. & 6.9 & 8.9 & 8.4 \\
Océanie & 1.1 & 0.7 & 0.9 & 0.9 & n.d. & 0.7 & 1.1 & 0.6 \\
Amérique du Sud & 2.4 & 2.8 & 2.4 & 2.4 & n.d. & 10.3 & 10.2 & 10.9 \\
Amérique centrale & & & & & & 2.2 & & \\
et Antilles & 3.0 & 3.4 & 2.6 & 3.2 & n.d. & & 2.4 & 5.4 \\
\hline
\end{tabular}

Source: Compilation à partir des données du Bureau de la Statistique du Québec.

de diversifier les partenaires commerciaux du Québec a modifié lâ nature de ses relations avec cette aire géographique.

L'étude des statistiques du commerce international nous permet de saisir l'évolution des échanges économiques du Québec avec l'Afrique de 1972 à $1981 .{ }^{13}$

Le tableau 1 révèle que parmi les grandes régions, l'Afrique se compare avantageusement avec l'Océanie (qui comprend l'Australie et la Nouvelle-Zélande), l'Asie (sans le Japon), l'Europe orientale (qui comprend l'URSS), l'Amérique centrale et les Antilles.

13. La compilation et la publication par les gouvernements des données statistiques du commerce international au niveau de désaggrégation utilisé dans nos tableaux se fait avec d'assez longs délais: ce n'est qu'au début de l'année 1984 que les données relatives au commerce international du Québec pour l'année 1982 furent disponibles. La structure du commerce international n'évoluant que très lentement, les données de 1982 sont aussi, dans l'ensemble, très près de celles de 1981, la dernière année reportée dans nos tableaux. 


\section{TABLEAU 2}

Exportations et Importations québécoises par régions d'Afrique pour les années $1972,1975,1978$ et 1981 .

(en pourcentage)

\begin{tabular}{|l|r|r|r|r||r|r|r|r|}
\hline & \multicolumn{4}{|c||}{ Exportations } & \multicolumn{4}{c|}{ Importations } \\
\cline { 2 - 9 } & 1972 & 1975 & 1978 & 1981 & 1972 & 1975 & 1978 & 1981 \\
\hline Afrique du Nord & 15.7 & 27.5 & 47.8 & 51.0 & n.d. & 12.1 & 43.6 & 70.2 \\
Afrique francophone & 17.0 & 16.2 & 19.2 & 20.0 & n.d. & 16.3 & 25.5 & 5.0 \\
Afrique anglophone & 45.7 & 38.0 & 23.0 & 20.6 & n.d. & 36.6 & 10.1 & 12.1 \\
Autres pays & 1.1 & 1.1 & 1.7 & 0.7 & n.d. & 0.5 & 1.0 & 0.1 \\
Afrique du Sud & 20.4 & 17.2 & 8.2 & 7.9 & n.d. & 34.4 & 20.4 & 12.6 \\
\hline
\end{tabular}

Source: Compilation à partir des données du Bureau de la Statistique du Québec.

Pour ce qui est des exportations, elle est plus importante que le Moyen-Orient. Sa contribution aux importations québécoises et canadiennes est restée relativement stable de 1972 à 1981 mais sa part en débouché pour nos produits a doublé.

Certains auteurs soutiennent que le commerce avec l'Afrique se réduit aux échanges avec l'Afrique du Sud et l'Algérie. Il est intéressant de regarder jusqu'à quel point les données par régions d'Afrique supportent un tel jugement.

On constate que l'Afrique du Nord est effectivement un partenaire privilégié pour le Québec (et pour le Canada). Le gain en importance de cette région a fait diminuer la part relative de l'Afrique anglophone et de l'Afrique du Sud même si ce dernier pays demeure toujours un partenaire très important. (tableau 2)

Outre l'Afrique du Nord, l'Afrique francophone a vu croître sa part des exportations québécoises (et canadiennes). On note également que toutes les régions ont perdu de l'importance au profit de l'Afrique du Nord. En 1981, l'Afrique du Nord comptait pour un peu plus de la moitié de tout le commerce québécois (et canadien) dans ce continent. Pour les autres régions, le commerce 
TABLEAU 3

Commerce international du Québec et du Canada avec l'Afrique pour les années 1972, 1975, 1978 et 1981 .

(en milliers de \$)

\begin{tabular}{|c|c|c|c|c|}
\hline & 1972 & 1975 & 1978 & 1981 \\
\hline \multicolumn{5}{|l|}{ Importations } \\
\hline Valeur pour le Canada & 275835 & 480944 & 442628 & 1118017 \\
\hline Valeur pour le Québec & n.d. & 317929 & 322996 & 687875 \\
\hline Part du Québec (\%) & n.d. & 66.1 & 72.9 & 57.9 \\
\hline \multicolumn{5}{|l|}{ Exportations } \\
\hline Valeur pour le Canada & 185007 & $4271+999$ & 636799 & 1557498 \\
\hline Valeur pour le Québec & 52673 & 99599 & 161850 & 503423 \\
\hline Part du Québec (\%) & 28.5 & 23.3 & 25.4 & 32.3 \\
\hline
\end{tabular}

Source: Compilation à partir des données du Bureau de la Statistique du Québec.

international du Québec et du Canada est sujet à de nombreuses fluctuations.

En désaggrégeant les données par pays, on peut déduire que les principaux partenaires du Québec et du Canada en Afrique sont parmi les plus développés de ce continent: Afrique du Sud, Nigéria, Algérie. Ceci est conforme à ce que nous avions suggéré en préambule à cette analyse à savoir que le Québec et le Canada se tournent vers les pays à «revenus moyens» du Tiers-Monde pour stimuler leurs exportations.

Le tableau 3 montre que le Québec joue un rôle de premier plan dans le développement des relations commerciales du Canada avec l'Afrique. La part du Québec dans les importations canadiennes est exceptionnellement élevée (de l'ordre de 65\%), mais ce taux ne reflète pas entièrement la réalité dans le cas des importations. ${ }^{14}$

14. Dans le cas particulier d'une province comme le Québec, les données sur les importations sont compilées selon les lieux de dédouanement (port, aéroport, etc.) et non selon la véritable origine ou l'ultime destination: ce sont donc des approximations 
Malgré tout, le tableau met en évidence l'apport du Québec dans le commerce canado-africain.

En somme, nous pouvons déduire d'une analyse du commerce international dans les années 70 que les intérêts privés québécois et canadiens ont bel et bien cherché à diversifier leurs partenaires commerciaux. Mais il appert que ce processus se fait très lentement et qu'il est surtout perceptible à partir du milieu de la décennie.

Les premières manifestations de cette tentative de diversification de nos échanges ont eu des répercussions sur nos relations traditionnelles avec l'Afrique. Ainsi ce continent qui, règle générale, est considéré comme un partenaire négligeable sur le plan commercial, a vu sa part de nos exportations doublée entre 1972 et 1981. Mais là où on obtient les résultats les plus probants, c'est lorsqu'on évalue pour le cas de l'Afrique, la stratégie (commune au Québec et au Canada) qui consiste à s'imposer sur les marchés des pays à "revenus moyens» du Tiers-Monde. L'augmentation substantielle de la part des exportations acheminées vers le Maghreb (surtout en Algérie) et dans une moindre mesure vers le Nigéria témoigne de cette volonté nouvelle.

Si le volet des échanges économiques est au centre des priorités du Québec et si les relations commerciales avec l'Afrique semblent être entrées dans une période de croissance, cela signifie-t-il que le domaine de l'aide ne constitue plus, aujourd'hui, une facette majeure des relations du Québec avec cette partie du monde?

Dans les faits, la coopération bilatérale du Québec avec l'Afrique a peu évolué ces dernières années: elle s'est manifestée par la poursuite des projets conjoints avec l'ACDI amorcés dans la période précédente et par sa participation à l'A.C.C.T. Le seul projet nouveau, ayant une certaine envergure, se résume à l'envoi

des véritables valeurs. Le rôle du Québec comme principal port d'entrée de l'est du Canada entraîne une distorsion des données d'importation. Dans le cadre du commerce international de la province, les données des exportations sont plus faibles. 
d'un fort contingent de professeurs de niveau C.E.G.E.P. au Maroc; cette action fut contrebalancée par le non-renouvellement de l'entente Québec-Maroc sur les frais de scolarité des étudiants, ressortissants de ce pays et fréquentant les universités québécoises.

Ce faible bilan de la coopération bilatérale ainsi que les préoccupations économiques maintes fois répétées du gouvernement peuvent laisser croire que l'aide et la coopération ne sont plus des volets majeurs de sa politique à l'égard de l'Afrique. Par ailleurs, les difficultés du Québec à occuper une place dans ce secteur ont été amplifiées par la redéfinition des politiques de l'ACDI.

Cette réorientation de l'aide canadienne se traduit dans son application par un nouveau mode de programmation de l'ACDI - l'action convergente - selon lequel l'agence planifie désormais le montant de l'assistance publique dispensée par le Canada au cours d'une période de cinq ans pour les pays bénéficiaires. Sans entrer dans les détails techniques que cette restructuration implique pour le mode de gestion et de fonctionnement de l'ACDI, il importe de savoir que ce chiffre indicatif inclut les sommes versée ou acheminées par l'entremise d'ONG et d'autres organismes du secteur privé en plus de celles consenties par le niveau gouvernemental. À toute fin pratique, cela signifie que l'ACDI essaie d'assumer la coordination (et le contrôle) de l'ensemble de l'aide canadienne pays par pays dans le but, semble-t-il d'obtenir des résultats plus probants.

En plus d'affecter le secteur privé de coopération, ce décloisonnement administratif de l'ACDI a également une incidence sur l'action future du gouvernement du Québec. Désormais, le Québec voit lui échapper le rôle de support logistique qu'il offrait aux universités et aux différentes corporations intéressées par une coopération avec leurs homologues africains. L'ACDI a mis sur pied un programme de coopération institutionnelle dont le but est de canaliser et de promouvoir la participation des institutions 
canadiennes dans le domaine de l'aide en leur fournissant les fonds nécessaires. Cette nouvelle dimension de la stratégie d'aide du Canada avec une volonté manifeste de contrôler toutes les actions canadiennes laissent peu de place à une intervention directe de la part du gouvernement du Québec.

Somme toute, la coopération bilatérale du Québec avec l'Afrique, si le gouvernement de la Province ne réagit pas promptement (et cela demeure improbable puisque ce volet ne constitue pas une des priorités du nouveau ministère du Commerce extérieur et des relations internationales), risque de régresser de façon critique, tendance qui est perceptible depuis la fin des années 70.

\section{5 - Conclusion}

À la suite du bilan que nous venons de dresser, que peuton envisager comme perspectives d'avenir pour les relations du Québec avec l'Afrique? L'état de nos recherches et certains actes posés récemment par le gouvernement du Québec font entrevoir un déplacement des préoccupations axées antérieurement sur une reconnaissance politique du Québec vers une rentabilisation en «termes économiques» de nos liens avec ce continent et avec les autres pays du Tiers-Monde.

Certains indices laissent supposer ce changement: la stratégie de l'action convergente de l'ACDI, l'échec du sommet francophone, la création du ministère du Commerce extérieur et de la Société d'exportation des ressources éducatives du Québec.

La nouvelle approche de l'ACDI n'accorde, pour ainsi dire, pas d'espace au Québec pour développer une coopération bilatérale avec l'Afrique. Cette omniprésence de l'ACDI et les contraintes financières évacuent à toute fin pratique le Québec de ce domaine, sauf s'il accepte d'y intervenir sous l'égide de l'organisme fédéral. 
Par ailleurs, la tactique que le Québec a toujours privilégié et qui consiste à être présent dans le monde de la francophonie, assuré qu'il était de l'appui de la France, peut à long terme lui être néfaste. La controverse qui entoure la tenue du sommet francophone où le Québec réclame un statut équivalent à celui qu'il a obtenu dans le cadre de l'A.C.C.T., montre la fragilité des acquis québécois. Il ne peut donc tabler indéfiniment sur son activité dans ces organismes pour être présent sur la scène internationale (d'autant plus que ce n'est un secret pour personne que ces organismes font preuve de peu d'efficacité et réunissent des pays n'ayant pas de véritables affinités historiques ou économiques).

L'ère des gains en terme strictement politique semble révolue au profit de celle de la rentabilité économique. Mais il est encore trop tôt pour en mesurer les effets tangibles sur les relations du Québec avec le continent africain. 